\title{
Darcy's Law for Flow in a Periodic Thin Porous Medium Confined Between Two Parallel Plates
}

\author{
John Fabricius ${ }^{1}$ • J. Gunnar I. Hellström² ${ }^{2}$ T. Staffan Lundström² \\ Elena Miroshnikova ${ }^{1}$. Peter Wall ${ }^{1}$
}

Received: 1 May 2015 / Accepted: 29 April 2016 / Published online: 12 May 2016

(C) The Author(s) 2016. This article is published with open access at Springerlink.com

\begin{abstract}
We study stationary incompressible fluid flow in a thin periodic porous medium. The medium under consideration is a bounded perforated $3 D$-domain confined between two parallel plates. The distance between the plates is $\delta$, and the perforation consists of $\varepsilon$ periodically distributed solid cylinders which connect the plates in perpendicular direction. Both parameters $\varepsilon, \delta$ are assumed to be small in comparison with the planar dimensions of the plates. By constructing asymptotic expansions, three cases are analysed: (1) $\varepsilon \ll \delta$, (2) $\delta / \varepsilon \sim$ constant and (3) $\varepsilon \gg \delta$. For each case, a permeability tensor is obtained by solving local problems. In the intermediate case, the cell problems are $3 D$, whereas they are $2 D$ in the other cases, which is a considerable simplification. The dimensional reduction can be used for a wide range of $\varepsilon$ and $\delta$ with maintained accuracy. This is illustrated by some numerical examples.
\end{abstract}

Keywords Thin porous media - Asymptotic analysis · Homogenization · Darcy's law · Mixed boundary condition $\cdot$ Stress boundary condition $\cdot$ Permeability

\section{Introduction}

There exist several mathematical approaches, collectively referred to as homogenization theory, for deriving Darcy's law (see e.g. Allaire 1989; Hornung 1997; Lions 1981; SanchezPalencia 1980; Tartar 1980 and the references therein), as well as methods based on phase averaging (Whitaker 1986). The present paper is devoted to deriving Darcy's law corresponding to incompressible viscous flow in a thin porous medium (TPM) by the multiscale expansion method which is a formal but powerful tool to analyse homogenization problems.

T. Staffan Lundström

staffan.lundstrom@1tu.se

1 Division of Mathematical Sciences, Luleå University of Technology, 97187 Luleå, Sweden

2 Division of Fluid and Experimental Mechanics, Luleå University of Technology,

97187 Luleå, Sweden 
The TPM considered involves two small parameters: the interspatial distance between obstacles $\varepsilon$ and the thickness of the domain $\delta$. More precisely, we consider pressure-driven flow through a periodic array of vertical cylinders confined between two parallel plates. The parallel plates make the geometry different from those studied in Chen and Papathanasiou (2008), Gebart (1992), Hellström et al. (2010), Hwang and Advani (2010), Jourak et al. (2013), Koch and Ladd (1997), Hellström et al. (2010), Lundström and Gebart (1995), Sangani and Yao (1988). A representative elementary volume for such TPM is a cube of lateral length $\varepsilon$ and vertical length $\delta$. The cube is repeated periodically in the space between the plates. Each cube can be divided into a fluid part and a solid part, where the solid part has the shape of a vertical cylinder (of length $\delta$ ). Hence the permeability of this TPM, denoted by $K^{\varepsilon \delta}$, depends on both $\varepsilon$ and $\delta$ as well as the geometry of the inclusions.

Pressure-driven flow within the plane of a confined thin porous medium takes place in a number of natural and industrial processes. This includes flow during manufacturing of fibre reinforced polymer composites with liquid moulding processes (Frishfelds et al. 2011; Nordlund and Lundström 2008; Tan and Pillai 2012), passive mixing in microfluidic systems (Jeon and Shin 2009) and paper making (Lundström et al. 2002; Singh et al. 2015).

Boundary value problems involving several small parameters are delicate to analyse as letting the parameters tend to zero at different rates may cause different asymptotic behaviour of the solutions. Therefore, one must distinguish three kinds of TPM whether $\varepsilon$ tends to zero slower, faster or at the same rate as $\delta$ :

VTPM The very thin porous medium is characterized by $\delta(\varepsilon) \ll \varepsilon$, i.e. the cylinder height is much smaller than the interspatial distance. The permeability tensor of VTPM satisfies $K^{\varepsilon \delta} \sim \delta^{2}(\varepsilon) K^{0}$ as $\varepsilon \rightarrow 0$, where $K^{0}$ depends only on the microgeometry.

PTPM The proportionally thin porous medium is characterized by $\delta(\varepsilon) / \varepsilon \sim \lambda$, where $\lambda$ is a positive constant. For example, this is the case when the cylinder height is proportional to the interspatial distance with $\lambda$ denoting the proportionality constant. The permeability tensor of PTPM satisfies $K^{\varepsilon \delta} \sim \delta^{2}(\varepsilon) K^{\lambda}$ as $\varepsilon \rightarrow 0$, where $K^{\lambda}$ depends on both $\lambda$ and the microgeometry.

HTPM The homogeneously thin porous medium is characterized by $\delta(\varepsilon) \gg \varepsilon$, i.e. the cylinder height is much larger than interspatial distance. The permeability tensor of HTPM satisfies $K^{\varepsilon \delta} \sim \varepsilon^{2} K^{\infty}$ as $\varepsilon \rightarrow 0$, where $K^{\infty}$ depends only on the microgeometry.

In all three cases, the asymptotic (or homogenized) pressure $p^{\lambda}$ is governed by a $2 D$ Darcy equation

$$
\nabla \cdot\left(K^{\lambda} \nabla p^{\lambda}\right)=0 \quad(0 \leq \lambda \leq \infty)
$$

satisfying a Dirichlet condition. The permeability tensor $K^{\lambda}$ is found by solving local boundary value problems, so-called cell problems, that involve neither $\varepsilon$ nor $\delta$. However, the local problems are different in each case. In the intermediate case (PTPM), the cell problems are three-dimensional and the coefficient of proportionality $\lambda$ appears as a parameter in the equations. In the extreme cases (VTPM and HTPM), the cell problems are two-dimensional, which is a considerable simplification compared with the intermediate case. VTPM and HTPM can also be considered as limiting cases of the intermediate case. Indeed, if (scaled) permeability $K^{\lambda}$ is regarded as a function of $\lambda$ and

$$
K^{0}=\lim _{\lambda \rightarrow 0} K^{\lambda}, \quad K^{\infty}=\lim _{\lambda \rightarrow \infty} \lambda^{2} K^{\lambda}
$$


then $K^{0}$ and $K^{\infty}$ are the permeabilities corresponding to VTPM and HTPM, respectively. This relation is confirmed theoretically both by constructing asymptotic expansions in $\lambda$ and by solving the cell problems numerically.

Mathematically the VTPM regime is analogous to flow in a Hele-Shaw cell. But this approximation is only valid for $\lambda \ll 1$, i.e. when the distance between the plates is much smaller than the interspatial distance between the obstacles. As $\lambda$ increases this approximation deviates more and more from the generic PTPM regime. Hele-Shaw flows have been studied by many authors, see e.g. Batchelor (1967), Saffman and Taylor (1958), Sherman (1990), Taylor (1967). For beautiful pictures of streamlines around obstacles between parallell plates, see the book Van Dyke (1982).

Flow past an array of circular cylindrical fibres confined between two parallel walls has been studied by Tsay and Weinbaum (1991), who extended a result obtained by Lee and Fung (1969). Their analysis is based on an approximate series solution of the Stokes equation. They claim that this solution describes the transition from the Hele-Shaw potential flow limit (corresponding to VTPM) to the viscous two-dimensional limiting case (corresponding to HTPM). However, their analysis does not give a distinct characterization of the PTPM regime, which is rigorously defined here. Moreover, their method is restricted to the particular geometry of circular cylinders, whereas our method can be applied to other geometries as well (see Remark 1).

\section{Preliminaries}

\subsection{Geometry of Media}

We consider flow in a thin domain which is perforated by periodically distributed vertical cylinders. In order to describe the geometry, precisely we introduce the following notation (which should be read together with Figs. 1, 2, 3). All 3D geometrical objects are denoted by bold font letters, whereas regular font letters are used for $2 D$ objects (Table 1).

\subsection{Differential Operators}

We consider fluid flow in the domain $\boldsymbol{\Omega}^{\varepsilon \delta}$. To have a domain that depends on neither $\varepsilon$ nor $\delta$, we shall reformulate the problem in the domain $\Omega \times Q^{f}$ by a change of variables.

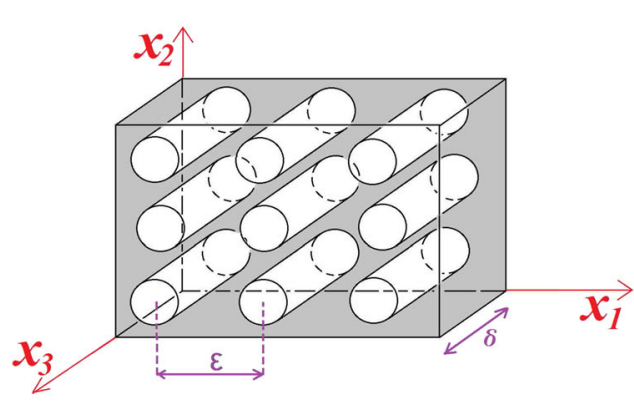

(a)

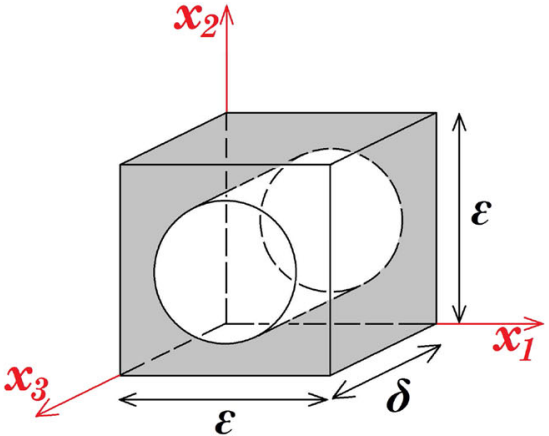

(b)

Fig. 1 Thin and perforated $3 D$ domain $\boldsymbol{\Omega}^{\varepsilon \delta}$. a $\boldsymbol{\Omega}^{\varepsilon \delta}$. b Representative elementary volume 


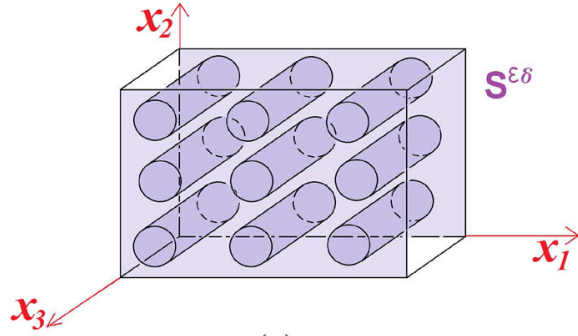

(a)

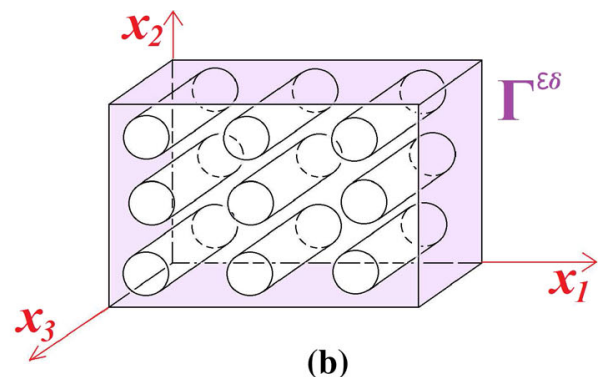

(b)

Fig. 2 Boundary $\partial \boldsymbol{\Omega}^{\varepsilon \delta}=\boldsymbol{S}^{\varepsilon \delta} \cup \boldsymbol{\Gamma}^{\varepsilon \delta}$. a $\boldsymbol{S}^{\varepsilon \delta}$. $\mathbf{b} \boldsymbol{\Gamma}^{\varepsilon \delta}$

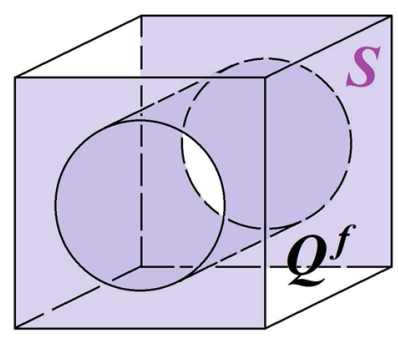

(a)

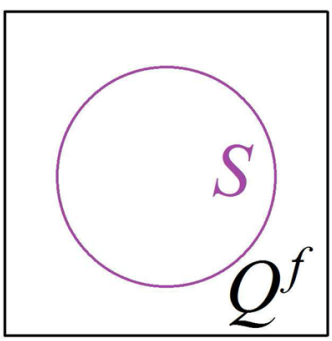

(b)

Fig. $33 D$ and $2 D$ unit cells. a $Q^{f}$. b $Q^{f}$

By convention, a point in $\Omega \times \boldsymbol{Q}^{f}$ is denoted by $\left(x_{1}, x_{2}, y_{1}, y_{2}, z\right)$, where $\left(x_{1}, x_{2}\right) \in \Omega$, $\left(y_{1}, y_{2}, z\right) \in \boldsymbol{Q}^{f}$. For the subsequent analysis, it is convenient to introduce abbreviations for various differential operators involving these variables.

Remark 1 The present analysis also holds true for any periodic arrangement of axial fibres of arbitrary cross-sectional shape. We have considered a square array of perpendicular cylinders for the sake of simplicity. However, it is possible to extend the analysis to inclined, curved or even crossing fibres. The main restriction is the assumption of periodicity (Tables 2, 3).

Remark 2 The superscript notation for domains and other variables should not be confused with exponents.

\subsection{Mathematical Model and Scaling of $\Omega^{\varepsilon \delta}$ into $\Omega^{\varepsilon}$}

An incompressible viscous fluid is well known to be described by the Navier-Stokes equations, coupled with boundary conditions of various types. We assume no-slip (Dirichlet) boundary condition on the solid boundary $S^{\varepsilon \delta}$ and a prescribed stress (Neumann) boundary condition on the lateral boundary $\boldsymbol{\Gamma}^{\varepsilon \delta}$. More precisely, we consider the Navier-Stokes system with a mixed boundary condition:

$$
\begin{cases}-\nabla P^{\varepsilon \delta}+v \Delta U^{\varepsilon \delta}=\left(U^{\varepsilon \delta} \cdot \nabla\right) U^{\varepsilon \delta} & \text { in } \boldsymbol{\Omega}^{\varepsilon \delta} \\ \nabla \cdot U^{\varepsilon \delta}=0 & \text { in } \boldsymbol{\Omega}^{\varepsilon \delta} \\ \left(-P^{\varepsilon \delta} I+v\left(\nabla U^{\varepsilon \delta}+\left(\nabla U^{\varepsilon \delta}\right)^{t}\right)\right) \boldsymbol{n}=-p^{b} \boldsymbol{n} & \text { on } \boldsymbol{\Gamma}^{\varepsilon \delta} \\ U^{\varepsilon \delta}=0 & \text { on } \boldsymbol{S}^{\varepsilon \delta}\end{cases}
$$


Table 1 Geometrical notation

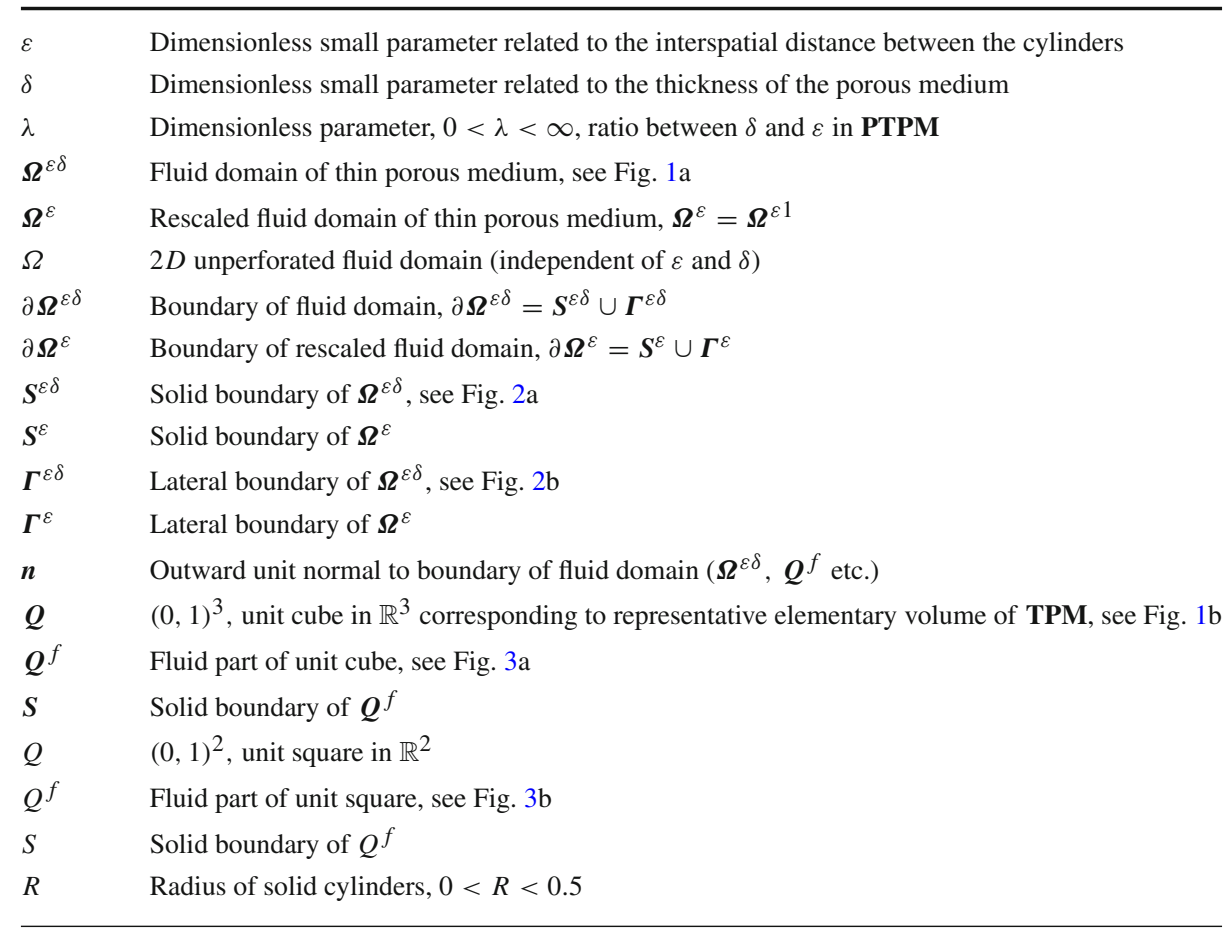

Table 2 Differential operators

\begin{tabular}{ll}
\hline$\nabla_{\delta}, \Delta_{\delta}$ & $\left(\frac{\partial}{\partial x_{1}}, \frac{\partial}{\partial x_{2}}, \frac{1}{\delta} \frac{\partial}{\partial z}\right), \frac{\partial^{2}}{\partial x_{1}^{2}}+\frac{\partial^{2}}{\partial x_{2}^{2}}+\frac{1}{\delta^{2}} \frac{\partial^{2}}{\partial z^{2}}$ \\
$\nabla_{x}, \Delta_{x}$ & $\left(\frac{\partial}{\partial x_{1}}, \frac{\partial}{\partial x_{2}}, 0\right), \frac{\partial^{2}}{\partial x_{1}^{2}}+\frac{\partial^{2}}{\partial x_{2}^{2}}$ \\
$\nabla_{y}, \Delta_{y}$ & $\left(\frac{\partial}{\partial y_{1}}, \frac{\partial}{\partial y_{2}}, 0\right), \frac{\partial^{2}}{\partial y_{1}^{2}}+\frac{\partial^{2}}{\partial y_{2}^{2}}$ \\
$\Delta_{x y}$ & $\frac{\partial^{2}}{\partial x_{1} \partial y_{1}}+\frac{\partial^{2}}{\partial x_{2} \partial y_{2}}$ \\
$\nabla_{z}, \Delta_{z}$ & $\left(0,0, \frac{\partial}{\partial z}\right), \frac{\partial^{2}}{\partial z^{2}}$ \\
$\nabla_{\lambda}, \Delta_{\lambda}$ & $\nabla_{y}+\frac{1}{\lambda} \nabla_{z}, \Delta_{y}+\frac{1}{\lambda^{2}} \Delta_{z}$ \\
\hline
\end{tabular}

where $v>0$ is a kinematic viscosity coefficient, $p^{b}: \Gamma^{\varepsilon \delta} \rightarrow \mathbb{R}$ is an external kinematic pressure which drives the flow in $\boldsymbol{\Omega}^{\varepsilon \delta}, P^{\varepsilon \delta}: \boldsymbol{\Omega}^{\varepsilon \delta} \rightarrow \mathbb{R}$ is the fluid kinematic pressure and $U^{\varepsilon \delta}: \boldsymbol{\Omega}^{\varepsilon \delta} \rightarrow \mathbb{R}^{3}$ is the fluid velocity (unknown functions). The function $p^{b}$ is also assumed to depend only on global variables $x_{1}, x_{2}$, ; hence, it is a macro characteristic of the flow.

As it mentioned before, the first step for studying (2) is to replace the domain $\boldsymbol{\Omega}^{\varepsilon \delta}$ with one that is independent of $\delta$. It can be easily done by changing variables $x_{3} \rightarrow x_{3} / \delta$. Under such rescaling, the domain $\boldsymbol{\Omega}^{\varepsilon \delta}$ is transformed into the domain $\boldsymbol{\Omega}^{\varepsilon}$ that has the same periodical structure in $x_{1}, x_{2}$-directions but with the unit length in $z=x_{3} / \delta$-direction. The boundary value problem (2) turns to the following one 
Table 3 Other symbols

\begin{tabular}{ll}
\hline$P^{\varepsilon \delta}, p^{\varepsilon \delta}$ & (Kinematic) pressure of incompressible fluid (see (2) and (3), respectively) \\
$p^{\lambda}$ & Homogenized pressure, $0 \leq \lambda \leq \infty$ \\
$p^{b}$ & Pressure on the lateral boundary \\
$U^{\varepsilon \delta}, u^{\varepsilon \delta}$ & Velocity field of incompressible fluid (see (2) and (3), respectively) \\
$u^{\lambda}$ & Homogenized velocity, $0 \leq \lambda \leq \infty$ \\
$v$ & Kinematic viscosity of incompressible fluid \\
$K^{\lambda}, K^{0}, K^{\infty}$ & Scaled permeability of PTPM $(0<\lambda<\infty)$, VTPM, HTPM, respectively \\
$k^{\lambda}, k^{0}, k^{\infty}$ & Diagonal elements of $K^{\lambda}, K^{0}, K^{\infty}$ in the case of isotropic permeability \\
$K^{\varepsilon \delta}$ & Permeability of TPM \\
$\left(W^{i}, q^{i}\right)$ & Solutions of cell problems, $i=1,2$ \\
\hline
\end{tabular}

$$
\begin{cases}-\nabla_{\delta} p^{\varepsilon \delta}+v \Delta_{\delta} u^{\varepsilon \delta}=\left(u^{\varepsilon \delta} \cdot \nabla_{\delta}\right) u^{\varepsilon \delta} & \text { in } \boldsymbol{\Omega}^{\varepsilon} \\ \nabla_{\delta} \cdot u^{\varepsilon \delta}=0 & \text { in } \boldsymbol{\Omega}^{\varepsilon} \\ \left(-p^{\varepsilon \delta} I+v\left(\nabla_{\delta} u^{\varepsilon \delta}+\left(\nabla_{\delta} u^{\varepsilon \delta}\right)^{t}\right)\right) \boldsymbol{n}=-p^{b} \boldsymbol{n} & \text { on } \boldsymbol{\Gamma}^{\varepsilon} \\ u^{\varepsilon \delta}=0 & \text { on } \boldsymbol{S}^{\varepsilon}\end{cases}
$$

where

$$
\begin{aligned}
& p^{\varepsilon \delta}\left(x_{1}, x_{2}, z\right)=P^{\varepsilon \delta}\left(x_{1}, x_{2}, \delta z\right), \\
& u^{\varepsilon \delta}\left(x_{1}, x_{2}, z\right)=U^{\varepsilon \delta}\left(x_{1}, x_{2}, \delta z\right),
\end{aligned} \quad\left(x_{1}, x_{2}, z\right) \in \boldsymbol{\Omega}^{\varepsilon} .
$$

Remark 3 As one can see, the flow in (2) is driven by the external pressure $p^{b}$ only. We would like to mention that it is also possible to include the force term in the first equation in (2), i.e. to consider

$$
-\nabla P^{\varepsilon \delta}+v \Delta U^{\varepsilon \delta}+f=\left(U^{\varepsilon \delta} \cdot \nabla\right) U^{\varepsilon \delta} \quad \text { in } \boldsymbol{\Omega}^{\varepsilon \delta},
$$

where $f: \boldsymbol{\Omega}^{\varepsilon \delta} \rightarrow \mathbb{R}^{3}$ as an external force acting on the unit mass of fluid, e.g. gravitational force.

\section{The Multiscale Asymptotic Expansion Method}

We seek a solution $\left(u^{\varepsilon \delta}, p^{\varepsilon \delta}\right)$ of (3) in the form of asymptotic expansions. The general idea of asymptotic expansions is to consider macro- and micro-behaviour of the solution separately, i.e. to suppose $x$ and $y=x / \varepsilon$ to be independent variables. Under such assumptions on $x$ and $y$, the unknown functions $u^{\varepsilon \delta}, p^{\varepsilon \delta}$ are presented as series with respect to small parameters $\delta$ and $\varepsilon$.

As it was shown in many classical papers (see e.g. Allaire 1989; Hornung 1997; Tartar 1980), if the velocity of flow is of 0 order (with respect to some small parameter of the domain geometry), then one should expect an extremely high fluid pressure, and on the contrary, for 0 order fluid pressure the corresponding flow is very slow. Since in our problem (2) the flow in governed by an external pressure $p^{b}$ which is independent of $\varepsilon$ and $\delta$ (in other words of 0 order), we assume the same order behaviour for unknown fluid pressure $p^{\varepsilon \delta}$. This allows us to start pressure series from 0 -order terms for both $\varepsilon$ and $\delta$ parameters and velocity series - from higher order terms. 
As announced in the introduction, three different flow regimes can be reached depending on the relation between $\varepsilon$ and $\delta$.

\subsection{Proportionally Thin Porous Medium (PTPM)}

Suppose that the thickness $\delta$ of the original domain $\boldsymbol{\Omega}^{\varepsilon \delta}$ is proportional to the size of inclusions $\varepsilon: \delta=\lambda \varepsilon$. Then we are looking for $u^{\varepsilon \delta}, p^{\varepsilon \delta}$ in the following form:

$$
\begin{aligned}
& u^{\varepsilon \delta}(x, z)=\sum_{i=2}^{\infty} \varepsilon^{i} \lambda^{i} u^{i}\left(x_{1}, x_{2}, \frac{x_{1}}{\varepsilon}, \frac{x_{2}}{\varepsilon}, z\right), \\
& p^{\varepsilon \delta}(x, z)=\sum_{j=0}^{\infty} \varepsilon^{j} \lambda^{j} p^{j}\left(x_{1}, x_{2}, \frac{x_{1}}{\varepsilon}, \frac{x_{2}}{\varepsilon}, z\right),
\end{aligned}
$$

where $\left(x_{1}, x_{2}\right) \in \Omega^{\varepsilon},\left(x_{1} / \varepsilon, x_{2} / \varepsilon, z\right)=\left(y_{1}, y_{2}, z\right) \in Q^{f}$ and functions $u^{i}, p^{j}, i=2,3, \ldots$, $j=0,1, \ldots$ are assumed to be the solution of the "extended" system (3) defined on the domain $\Omega \times Q^{f}$ :

$$
\begin{cases}-\sum_{i=0}^{\infty} \varepsilon^{i} \lambda^{i}\left(\nabla_{x}+\frac{1}{\varepsilon} \nabla_{\lambda}\right) p^{i} & \\ +v \sum_{i=2}^{\infty} \varepsilon^{i} \lambda^{i}\left(\Delta_{x}+\frac{2}{\varepsilon} \Delta_{x y}+\frac{1}{\varepsilon^{2}} \Delta_{\lambda}\right) u^{i} & \text { in } \Omega \times \boldsymbol{Q}^{f} \\ =\sum_{i=2}^{\infty} \varepsilon^{i} \lambda^{i}\left(u^{i} \cdot\left(\nabla_{x}+\frac{1}{\varepsilon} \nabla_{\lambda}\right)\right) \sum_{j=2}^{\infty} \varepsilon^{j} \lambda^{j} u^{j} & \\ \sum_{i=2}^{\infty} \varepsilon^{i} \lambda^{i}\left(\nabla_{x}+\frac{1}{\varepsilon} \nabla_{\lambda}\right) \cdot u^{i}=0 & \text { in } \Omega \times \boldsymbol{Q}^{f} \\ \sum_{i=2}^{\infty} \varepsilon^{i} \lambda^{i} u^{i}=0 & \text { on } \Omega \times S \\ v \sum_{i=2}^{\infty} \varepsilon^{i} \lambda^{i}\left(\left(\nabla_{x} u^{i}+\left(\nabla_{x} u^{i}\right)^{t}\right)+\frac{1}{\varepsilon}\left(\nabla_{\lambda} u^{i}+\left(\nabla_{\lambda} u^{i}\right)^{t}\right)\right) & \\ -\sum_{i=0}^{\infty} \varepsilon^{i} \lambda^{i} p^{i} I=-p^{b} I & \text { on } \partial \Omega \times Q^{f}\end{cases}
$$

Due to periodicity of $\omega$, another natural assumption on $u^{i}, p^{j}, i=2,3, \ldots, j=0,1, \ldots$, is to suppose them to be 1-periodic with respect to $y$.

All further results are based on collecting terms in (5) with equal powers of $\varepsilon$. For the momentum equation, we have

$$
\begin{aligned}
\varepsilon^{-1} & : \nabla_{\lambda} p^{0}=0, \\
\varepsilon^{0} & :-\left(\nabla_{x} p^{0}+\lambda \nabla_{\lambda} p^{1}\right)+\nu \lambda^{2} \Delta_{\lambda}\left(u^{2}\right)=0,
\end{aligned}
$$

and

$$
\begin{aligned}
& \varepsilon^{1}: \nabla_{\lambda} \cdot u^{2}=0, \\
& \varepsilon^{2}: \nabla_{x} \cdot u^{2}+\lambda \nabla_{\lambda} \cdot u^{3}=0,
\end{aligned}
$$


for the conservation of mass (here we have divided equations by $\lambda^{2}$ ). Boundary conditions provide the following

$$
u^{i}=0 \text { on } \Omega \times S, \quad i=0,1, \ldots
$$

for the boundary on micro-scale and

$$
\begin{gathered}
p^{0}=p^{b} \text { on } \partial \Omega \times \boldsymbol{Q}^{f}, \\
-p^{1} I+v \lambda\left(\nabla_{\lambda} u^{2}+\left(\nabla_{\lambda} u^{2}\right)^{t}\right)=0 \quad \text { on } \partial \Omega \times \boldsymbol{Q}^{f},
\end{gathered}
$$

for the global boundary.

Remark 4 Note that the inertial term does not appear in Eqs. (6a-9b). Inertial effects may be included by taking higher order terms into account or by choosing a different scaling of the problem.

Thus (6a) implies that $p^{0}$ is a function of $x$ alone, i.e. $p^{0}=p^{0}(x)$, and satisfies (9a) on $\partial \Omega$. From (6b), (7a), (8) and (9b), we get the next system:

$$
\begin{cases}-\frac{1}{\lambda^{2}} \nabla_{x} p^{0}-\frac{1}{\lambda} \nabla_{\lambda} p^{1}+v \Delta_{\lambda}\left(u^{2}\right)=0 & \text { in } \Omega \times \boldsymbol{Q}^{f}, \\ \nabla_{\lambda} \cdot u^{2}=0 & \text { in } \Omega \times \boldsymbol{Q}^{f}, \\ u^{2}=0 & \text { on } \Omega \times \boldsymbol{S}, \\ -p^{1} I+v \lambda\left(\nabla_{\lambda} u^{2}+\left(\nabla_{\lambda} u^{2}\right)^{t}\right)=0 & \text { on } \partial \Omega \times \boldsymbol{Q}^{f} .\end{cases}
$$

Taking into account that $p^{0}$ does not depend on $(y, z) \in Q^{f}$, we can write $u^{2}, p^{1}$ as a linear combinations

$$
\begin{aligned}
& u^{2}(x, y, z)=\frac{1}{v} \sum_{i=1}^{2} \frac{\partial p^{0}(x)}{\partial x_{i}} W^{i}(y, z), \\
& p^{1}(x, y, z)=\sum_{i=1}^{2} \frac{\partial p^{0}(x)}{\partial x_{i}} q^{i}(y, z),
\end{aligned}
$$

where $\left(W^{i}, q^{i}\right), i=1,2,3$, are 1-periodic (in $Q^{f}$ ) solutions of the following cell problems:

$$
\begin{cases}-\frac{1}{\lambda} \nabla_{\lambda} q^{i}+\Delta_{\lambda} W^{i}-\frac{1}{\lambda^{2}} e^{i}=0 & \text { in } \boldsymbol{Q}^{f}, \\ \nabla_{\lambda} \cdot W^{i}=0 & \text { in } \boldsymbol{Q}^{f}, \\ W^{i}=0 & \text { on } \boldsymbol{S} .\end{cases}
$$

Here $e_{i}=\left(\delta_{1 i}, \delta_{2 i}, 0\right), i=1,2,3$, and $\delta_{j i}$ is the Kronecker delta. One can see that $W^{3}=0$, $q^{3}=$ const since for $i=3$ the force term is absent.

Substitution of (11) into (7b) and integration of it with respect to $(y, z)$ over $\boldsymbol{Q}^{f}$ (with additional factor $1 /|\boldsymbol{Q}|$ ) provide Darcy's law (the term with $\nabla_{\lambda} \cdot u^{3}$ vanishes because of periodicity of $u^{3}$ with respect to $y$ and since $u^{3}=0$ on $S$ ):

$$
\begin{aligned}
& \frac{1}{|\boldsymbol{Q}|} \int_{\boldsymbol{Q}^{f}}\left(\nabla_{x} \cdot u^{2}+\lambda \nabla_{\lambda} \cdot u^{3}\right) \mathrm{d} y \mathrm{~d} z=\frac{1}{|\boldsymbol{Q}|} \int_{\boldsymbol{Q}^{f}} \nabla_{x} \cdot u^{2} \mathrm{~d} y \mathrm{~d} z \\
& \quad=\frac{1}{v} \frac{1}{|\boldsymbol{Q}|} \int_{\boldsymbol{Q}^{f}} \nabla_{x} \cdot\left(\sum_{i=1}^{2} \frac{\partial p^{0}(x)}{\partial x_{i}} W^{i}(y, z)\right) \mathrm{d} y \mathrm{~d} z \\
& \quad=\frac{1}{v} \nabla_{x} \cdot\left(K^{\lambda}\left(\nabla_{x} p^{0}\right)\right)=0,
\end{aligned}
$$


where $K^{\lambda}$ is the permeability matrix $3 \times 3$ with components given by the expression

$$
K_{i j}^{\lambda}=\frac{1}{|\boldsymbol{Q}|} \int_{\boldsymbol{Q}^{f}} W_{j}^{i} \mathrm{~d} y \mathrm{~d} z, \quad W^{i}=\left(W_{1}^{i}, W_{2}^{i}, W_{3}^{i}\right), \quad i, j=1,2,3 .
$$

By multiplying (12) with $W^{j}$ and integrating by parts, one deduces the equivalent expression for the permeability:

$$
K_{i j}^{\lambda}=-\frac{\lambda^{2}}{|\boldsymbol{Q}|} \int_{\boldsymbol{Q}^{f}} \nabla_{\lambda} W^{i}: \nabla_{\lambda} W^{j} \mathrm{~d} y \mathrm{~d} z, \quad i, j=1,2,3 .
$$

In particular, this implies that $K^{\lambda}$

$$
K^{\lambda}=\left(\begin{array}{ccc}
k^{\lambda} & 0 & 0 \\
0 & k^{\lambda} & 0 \\
0 & 0 & 0
\end{array}\right)
$$

In further asymptotic analysis, we will concentrate on the form of the permeability matrix $K^{\lambda}$. This is due to the fact that the values for elements of $K^{\lambda}$ are verified by numerical calculations in Sect. 3.

\subsection{Limit Cases}

In this section, two different approaches for analysing VTPM and HTPM are presented.

\subsubsection{Asymptotic $\lambda$-Analysis $(\lambda \rightarrow 0)$}

To pass to the limit $\lambda \rightarrow 0$, we start from (12)

$$
\begin{cases}-\frac{1}{\lambda} \nabla_{\lambda} q^{i}+\Delta_{\lambda} W^{i}-\frac{1}{\lambda^{2}} e^{i}=0 & \text { in } \boldsymbol{Q}^{f}, \\ \nabla_{\lambda} \cdot W^{i}=0 & \text { in } \boldsymbol{Q}^{f}, \quad i=1,2,3 . \\ W^{i}=0 & \text { on } \boldsymbol{S},\end{cases}
$$

By changing a variable $z \rightarrow \lambda z, z \in(0,1)$, one can see that the unit domain $\boldsymbol{Q}^{f}$ is transferred to the thin cell $Q^{f} \times(0, \lambda)$ with $\lambda \rightarrow 0$ and for $Q^{f} \times(0, \lambda)$ the corresponding momentum equation has the following form

$$
-\frac{1}{\lambda} \nabla q^{i}+\Delta W^{i}-\frac{1}{\lambda^{2}} e^{i}=0
$$

So in fact we deal now with a thin domain and because of it in this section we will use lower limits different from previous. As one can see from (18), the magnitude of viscous forces $\lambda^{-2} e^{i}$ is proportional to $\lambda^{-2}$, then the fluid pressure is assumed to balance viscous force, and now we are looking for the solutions in the following form

$$
\begin{aligned}
W^{i}(y, z) & =\sum_{j=0}^{\infty} \lambda^{j} w^{i, j}\left(y_{1}, y_{2}, z\right), \\
q^{i}(y, z) & =\sum_{j=-2}^{\infty} \lambda^{j} q^{i, j}\left(y_{1}, y_{2}, z\right),
\end{aligned}
$$


As before all functions $w^{i, j}, q^{i, j}$ are assumed to be 1-periodic in the $y$-directions and to satisfy the next problem (we multiply the momentum equation with $\lambda^{2}$ for the simplicity):

$$
\begin{cases}-\sum_{j=-2}^{\infty} \lambda^{j+1}\left(\nabla_{y}+\frac{1}{\lambda} \nabla_{z}\right) q^{i, j}+ & \text { in } \boldsymbol{Q}^{f}, \\ +\sum_{j=0}^{\infty} \lambda^{j+2}\left(\Delta_{y}+\frac{1}{\lambda^{2}} \Delta_{z}\right) w^{i, j}-e^{i}=0 & \\ \sum_{j=0}^{\infty} \lambda^{j}\left(\nabla_{y}+\frac{1}{\lambda} \nabla_{z}\right) \cdot w^{i, j}=0 & \text { in } \boldsymbol{Q}^{f}, \\ \sum_{j=0}^{\infty} \lambda^{j} w^{i, j}=0 & \text { on } S,\end{cases}
$$

Recall that $q^{3}$ and $W^{3}$ vanish in $\boldsymbol{Q}^{f}$. Then expansions for $i=3$ in (19) are trivial, and all terms $w^{3, j}, q^{3, j}$ are also assumed to be zero. So further analysis should be considered mostly for $i=1,2$ (for $i=3$ it is also valid, but all solutions are trivial again).

For different powers of $\lambda$ in the momentum equation, we get

$$
\begin{aligned}
& \lambda^{-2}: \nabla_{z} q^{i,-2}=0, \\
& \lambda^{-1}: \nabla_{y} q^{i,-2}+\nabla_{z} q^{i,-1}=0, \\
& \lambda^{0}:-\left(\nabla_{y} q^{i,-1}+\nabla_{z} q^{i, 0}\right)+\Delta_{z}\left(w^{i, 0}\right)-e^{i}=0,
\end{aligned}
$$

and

$$
\begin{aligned}
& \lambda^{-1}: \nabla_{z} \cdot w^{i, 0}=0, \\
& \lambda^{0}: \nabla_{y} \cdot w^{i, 0}+\nabla_{z} \cdot w^{i, 1}=0,
\end{aligned}
$$

for the conservation of mass. The last equation in (20) implies

$$
w^{i, j}=0 \quad \text { on } \Omega \times S, \quad j=0,1, \ldots
$$

Such collecting terms with equal powers of $\lambda$ provide the following results:

$-w_{3}^{i, 0}=0$ (from (22a) and (23)).

$-q^{i,-2}=$ const and $q^{i,-1}=q^{i,-1}(y)$, (from (21a) and (21b)).

For the third component in (21c), we have $q^{i, 0}=q^{i, 0}(y)$, ( since $w_{3}^{i, 0}=0$ ). Taking these facts and boundary condition for $w^{i, 0}$ into account, by integration of $(21 \mathrm{c})$ we get

$$
w^{i, 0}(y, z)=\frac{z(z-1)}{2}\left(\nabla_{y} q^{i,-1}(y)+e^{i}\right), \quad(y, z) \in Q^{f} .
$$

Integrating $(22 \mathrm{~b})$ over $(0,1)$, we also obtain

$$
\begin{aligned}
& \int_{(0,1)} \nabla_{y} \cdot \sum_{j=1}^{2}\left(\nabla_{y} q^{i,-1}(y)+e^{i}\right) \frac{z(z-1)}{2} \mathrm{~d} z=\frac{1}{12} \sum_{j=1}^{2} \nabla_{y} \cdot\left(\nabla_{y} q^{i,-1}(y)+e^{i}\right) \\
& =\frac{1}{12} \nabla_{y} \cdot\left(e^{i}+\nabla_{y} q^{i,-1}\right)=\frac{1}{12} \Delta_{y} q^{i,-1}=0 .
\end{aligned}
$$


To "extract" boundary conditions, we multiply $\nabla_{y} \cdot\left(e^{i}+\nabla_{y} q^{i,-1}\right)=0$ by an arbitrary periodic divergence-free vector function $\varphi: Q^{f} \rightarrow \mathbb{R}^{3}$ vanishing on $S$ and integrate over $Q^{f}$ :

$$
\begin{aligned}
0= & \int_{Q^{f}} \nabla_{y} \cdot\left(e^{i}+\nabla_{y} q^{i,-1}\right) \varphi \mathrm{d} y=\int_{S}\left(e^{i}+\nabla_{y} q^{i,-1}\right) \varphi \boldsymbol{n} \mathrm{d} S \\
& -\int_{Q^{f}}\left(\nabla_{y} q^{i,-1}+e^{i}\right) \nabla_{y} \cdot \varphi \mathrm{d} y=0,
\end{aligned}
$$

due to periodicity of $\varphi$ we get

$$
\left(e^{i}+\nabla_{y} q^{i,-1}\right) \cdot \boldsymbol{n}=0 \quad \text { on } S .
$$

Returning to (15) and using (24), we obtain the final expression for the permeability matrix $K^{0}$

$$
K_{i j}^{0}=\frac{1}{|\boldsymbol{Q}|} \int_{\boldsymbol{Q}^{f}} w_{j}^{i, 0} \mathrm{~d} y \mathrm{~d} z=\frac{1}{12|\boldsymbol{Q}|} \int_{Q^{f}}\left(\nabla_{y} q^{i,-1}+e^{i}\right) \cdot e^{j} \mathrm{~d} y, \quad i=1,2,3,
$$

where $q^{i,-1}, i=1,2$, are the 1 -periodic solutions of the problem

$$
\begin{cases}\Delta_{y} q^{i,-1}=0 & \text { in } Q^{f} \\ \left(\nabla_{y} q^{i,-1}+e^{i}\right) \cdot \boldsymbol{n}=0 & \text { on } S\end{cases}
$$

We recall again that $q^{3,-1}=0$.

Remark 5 Taking into account that the first non-vanishing term in the asymptotic expansion for the velocity $W^{i}$ in (19) is of the order $\lambda^{0}$, we can conclude from comparison of (15) and (25) that

$$
K^{\lambda} \sim K^{0} \quad \text { as } \lambda \rightarrow 0,
$$

where

$$
K^{0}=\left(\begin{array}{ccc}
k^{0} & 0 & 0 \\
0 & k^{0} & 0 \\
0 & 0 & 0
\end{array}\right)
$$

\subsubsection{Asymptotic $\lambda$-Analysis $(\lambda \rightarrow \infty)$}

For this case, we have $1 / \lambda$ tending to zero. To use the same technique with respect to a small parameter, let us introduce $\sigma=1 / \lambda \rightarrow 0$. After such changes for (12), we obtain

$$
\begin{cases}-\frac{1}{\sigma}\left(\nabla_{y}+\sigma \nabla_{z}\right) q^{i}+\frac{1}{\sigma^{2}}\left(\Delta_{y}+\sigma^{2} \Delta_{z}\right) W^{i}-e_{i}=0 & \text { in } \boldsymbol{Q}^{f}, \\ \left(\nabla_{y}+\sigma \nabla_{z}\right) \cdot W^{i}=0 & \text { in } \boldsymbol{Q}^{f}, \\ W^{i}=0 & \text { on } \boldsymbol{S},\end{cases}
$$

We use the following 1-periodic (in $y$-directions) series for $q^{i}$ and $W^{i}, i=1,2$ :

$$
\begin{aligned}
W^{i}(y, z) & =\sum_{j=2}^{\infty} \sigma^{j} w^{i, j}\left(y_{1}, y_{2}, z\right), \\
q^{i}(y, z) & =\sum_{j=0}^{\infty} \sigma^{j} q^{i, j}\left(y_{1}, y_{2}, z\right),
\end{aligned}
$$


As it was mentioned in previous section, all corresponding terms $w^{3, j}, q^{3, j}$ are trivial.

By substituting (30) into (29), we obtain

$$
\begin{aligned}
\sigma^{-1} & : \nabla_{y} q^{i, 0}=0, \\
\sigma^{0} & :-\left(\nabla_{y} q^{i, 1}+\nabla_{z} q^{i, 0}\right)+\Delta_{y} w^{i, 2}-e^{i}=0,
\end{aligned}
$$

and

$$
\begin{aligned}
& \sigma^{1}: \nabla_{y} \cdot w^{i, 2}=0, \\
& \sigma^{3}: \nabla_{y} \cdot w^{i, 2}+\nabla_{z} \cdot w^{i, 3}=0,
\end{aligned}
$$

for the first and second equations in (29). Boundary conditions are

$$
w^{i, j}=0 \text { on } S \quad \forall j=2, \ldots
$$

From (31a), we have that $q^{i, 0}$ does not depend on $y \in Q^{f}$.

By integration of (32b) over $Q^{f}$, we get $\frac{\partial}{\partial z} \int_{Q^{f}} w_{3}^{i, 2} \mathrm{~d} y=\int_{Q^{f}} \frac{\partial w_{3}^{i, 2}}{\partial z} \mathrm{~d} y=0$, but we can also integrate with respect to $z$ :

$$
\int_{0}^{z} \int_{Q^{f}} \frac{\partial w_{3}^{i, 2}}{\partial z} \mathrm{~d} y \mathrm{~d} z=\int_{Q^{f}} w_{3}^{i, 2} \mathrm{~d} y=0 .
$$

So, $w_{3}^{i, 2}$ has zero mean value.

Write (31b) in componentwise form:

$$
-\left(\begin{array}{c}
\frac{\partial q^{i, 1}}{\partial y_{1}} \\
\frac{\partial q^{i, 1}}{\partial y_{2}} \\
\frac{\partial q^{i, 0}}{\partial z}
\end{array}\right)+\left(\begin{array}{c}
\frac{\partial^{2} w_{1}^{i, 2}}{\partial y_{1}^{2}}+\frac{\partial^{2} w_{1}^{i, 2}}{\partial y_{2}^{2}} \\
\frac{\partial^{2} w_{2}^{i, 2}}{\partial y_{1}^{2}}+\frac{\partial^{2} w_{2}^{i, 2}}{\partial y_{2}^{2}} \\
\frac{\partial^{2} w_{3}^{i, 2}}{\partial y_{1}^{2}}+\frac{\partial^{2} w_{3}^{i, 2}}{\partial y_{2}^{2}}
\end{array}\right)-e^{i}=\left(\begin{array}{l}
0 \\
0 \\
0
\end{array}\right) .
$$

Regarding the third component, we multiply it by an arbitrary periodic (with respect to $y$ ) function $\phi$ which has zero mean value and integrate with respect to $y$ by parts. It provides us

$$
\int_{Q^{f}}\left(-\nabla_{z} q^{i, 0}+\Delta_{y} w_{3}^{i, 2}\right) \phi \mathrm{d} y=-\int_{Q^{f}} \nabla_{y} w_{3}^{i, 2}: \nabla_{y} \phi \mathrm{d} y=0,
$$

since $q^{i, 0}=q^{i, 0}(z)$. By substituting $\phi=w_{3}^{i, 2}$ and using boundary condition for $w^{i, 2}$ on $\boldsymbol{S}$, we get

$$
w_{3}^{i, 2}=0
$$

For the first two components, we have

$$
\begin{cases}-\nabla_{y} q^{i, 1}+\Delta_{y} w^{i, 2}-e^{i}=0 & \text { in } \boldsymbol{Q}^{f} \\ \nabla_{y} \cdot w^{i, 2}=0 & \text { in } \boldsymbol{Q}^{f} \\ w^{i, 2}=0 & \text { on } \boldsymbol{S}\end{cases}
$$


Also there is no $z$-dependence in the system above, then it is correct to consider all equations in 2D-domain $Q^{f}$ (instead of $\boldsymbol{Q}^{f}$ ). Finally for the permeability (15) we have

$$
K_{i j}^{\infty}=\frac{1}{|Q|} \int_{Q^{f}} w^{i, 2} \cdot e^{j} \mathrm{~d} y, \quad i, j=1,2,3,
$$

where $w^{i, 2}, i=1,2$, are the solutions of

$$
\begin{cases}-\nabla_{y} q^{i, 1}+\Delta_{y} w^{i, 2}-e^{i}=0 & \text { in } Q^{f} \\ \nabla_{y} \cdot w^{i, 2}=0 & \text { in } Q^{f} \\ w^{i, 2}=0 & \text { on } S\end{cases}
$$

and $w^{3,2}=0$ again.

Remark 6 Since the first non-vanishing term in $\sigma$-expansions for the velocity $W^{i}$ in (30) is of the order $\sigma^{2}=\lambda^{-2}$, from the corresponding expressions (15) and (35) for the permeability we obtain

$$
K^{\lambda} \sim \frac{1}{\lambda^{2}} K^{\infty} \quad \text { as } \lambda \rightarrow \infty
$$

where

$$
K^{\infty}=\left(\begin{array}{ccc}
k^{\infty} & 0 & 0 \\
0 & k^{\infty} & 0 \\
0 & 0 & 0
\end{array}\right)
$$

\subsubsection{Very Thin Porous Medium (VTPM)}

The case $\delta \ll \varepsilon$ can be modelled e.g. by the relation $\delta=\varepsilon^{2}$. Since $\delta$ is a function of $\varepsilon$, we simply write $u^{\varepsilon}=u^{\varepsilon \delta}$ and $p^{\varepsilon}=p^{\varepsilon \delta}$. We choose the following series representation for the solution of (3)

$$
\begin{aligned}
& u^{\varepsilon}(x, z)=\sum_{i=4}^{\infty} \varepsilon^{i} u^{i}\left(x_{1}, x_{2}, \frac{x_{1}}{\varepsilon}, \frac{x_{2}}{\varepsilon}, z\right), \\
& p^{\varepsilon}(x, z)=\sum_{i=0}^{\infty} \varepsilon^{i} p^{i}\left(x_{1}, x_{2}, \frac{x_{1}}{\varepsilon}, \frac{x_{2}}{\varepsilon}, z\right),
\end{aligned}
$$

where $\left(x_{1}, x_{2}\right) \in \Omega^{\varepsilon},\left(\frac{x_{1}}{\varepsilon}, \frac{x_{2}}{\varepsilon}, z\right)=(y, z) \in Q^{f}$.

By the same method as before, we come to the following conclusions:

$-p^{0}=p^{0}(x), p^{1,2}=p^{1,2}(x, y), u_{3}^{4}=0 ;$

$-u_{1,2}^{4}(x, y, z)=\frac{z(z-1)}{2 v} \sum_{i=1}^{2} \nabla_{x} p^{0}(x)\left(\nabla_{y} q^{i}+e^{i}\right),(x, y, z) \in \Omega \times \boldsymbol{Q}^{f}$, where

$$
\begin{cases}\Delta_{y} q^{i, 2}=0 & \text { in } Q^{f} \\ \left(\nabla_{y} q^{i, 2}+e^{i}\right) \cdot \boldsymbol{n}=0 & \text { on } S .\end{cases}
$$

- Darcy's law has the following form:

$$
\nabla_{x} \cdot\left(K^{0}\left(\nabla_{x} p^{0}\right)\right)=0,
$$

where

$$
K^{0}=\frac{1}{12|Q|} \int_{Q^{f}}\left(\begin{array}{ccc}
1+\frac{\partial q^{1}}{\partial y_{1}} & \frac{\partial q^{2}}{\partial y_{1}} & 0 \\
\frac{\partial q^{1}}{\partial y_{2}} & 1+\frac{\partial q^{2}}{\partial y_{2}} & 0 \\
0 & 0 & 0
\end{array}\right) \mathrm{d} y
$$


is the permeability matrix.

Remark 7 One can see that the difference between the lowest $\varepsilon$ limits for pressure and velocity series in (39) is of four orders instead of two (compare with (4), (19), (30)). But let us note that in this case $\varepsilon$ is not the smallest parameter. In terms of $\delta$ (now the smallest one), the difference is still of two orders ( since $\varepsilon^{4}=\delta^{2}$ ).

Remark 8 Another important moment is the scale of real permeability. Since in (39) the lowest term in the velocity expansions is of the order $\varepsilon^{4}=\delta^{2}$, then to obtain the real value of permeability, the matrix $K^{0}$ should be scaled by factor $\delta^{2}$.

\subsubsection{Homogeneously Thin Porous Medium (HTPM)}

To model case $\delta \gg \varepsilon$, we can suppose e.g. that the square of the thickness $\delta$ of $\boldsymbol{\Omega}^{\varepsilon \delta}$ is proportional to $\varepsilon: \varepsilon=\delta^{2}$. Since $\varepsilon$ is a function of $\delta$, we simply write $u^{\delta}=u^{\varepsilon \delta}$ and $p^{\delta}=p^{\varepsilon \delta}$.

We consider the following series:

$$
u^{\delta}(x, z)=\sum_{i=4}^{\infty} \delta^{i} u^{i}\left(x_{1}, x_{2}, \frac{x_{1}}{\delta^{2}}, \frac{x_{2}}{\delta^{2}}, z\right), \quad p^{\delta}(x, z)=\sum_{i=0}^{\infty} \delta^{i} p^{i}\left(x_{1}, x_{2}, \frac{x_{1}}{\delta^{2}}, \frac{x_{2}}{\delta^{2}}, z\right),
$$

where $\left(x_{1}, x_{2}\right) \in \Omega^{\varepsilon},\left(x_{1} / \delta^{2}, x_{2} / \delta^{2}, z\right)=(y, z) \in \boldsymbol{Q}^{f}$.

All further manipulations are similar to those which were done in Sect. 2.2.2. Finally, we obtain

$-p^{0,1}=p^{0,1}(x), u_{3}^{4}=0$;

- $u_{1,2}^{4}=u_{1,2}^{4}(x, y), p^{2}=p^{2}(x, y)$;

- $u^{4}(x, y)=\frac{1}{v} \sum_{i=1}^{2} \nabla_{x} p^{0} W^{i}(y), p^{2}(x, y)=\sum_{i=1}^{2} \nabla_{x} p^{0} q^{i}(y)$ in $\Omega \times Q^{f}$, where $\left(W^{i}, q^{i}\right)$,

$i=1,2$ are solutions of cell problem

$$
\begin{cases}-e^{i}-\nabla_{y} q^{i}+\Delta_{y} W^{i}=0 & \text { in } Q^{f}, \\ \nabla_{y} \cdot W^{i}=0 & \text { in } Q^{f}, \\ W^{i}=0 & \text { on } S\end{cases}
$$

and $\left(W^{3}, q^{3}\right)=(0,0)$. Originally system (43) was defined in $\boldsymbol{Q}^{f}$, but one can easy see that equations above do not depend on $z$. It means that their solutions are independent of $z$ also and problem (43) can be considered only for the flat domain $Q^{f}$ without any contradiction.

- the Darcy's law can be written in the next form

$$
\frac{1}{v} \nabla_{x} \cdot\left(K^{\infty} \nabla_{x} p^{0}\right)=0,
$$

where

$$
K_{i j}^{\infty}=\frac{1}{|Q|} \int_{Q^{f}} W_{j}^{i} \mathrm{~d} y, \quad i, j=1,2,3 .
$$

By using the similar argumentation as it was presented in previous section (see Remarks 7 , 8 ), we would like to mention that the difference between the lowest terms for pressure and velocity in (42) is still of two orders with respect to the smallest parameter (which is $\varepsilon$ now) and that the permeability for the real problem is $\varepsilon^{2} K^{\infty}$. 


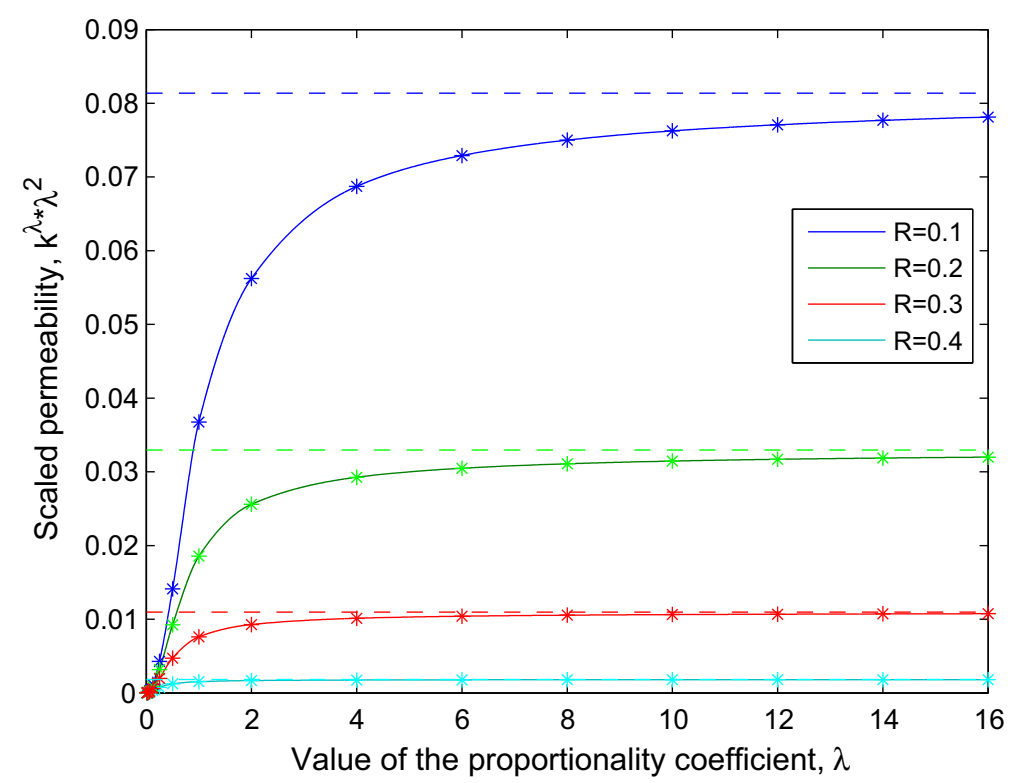

Fig. $4 \lambda^{2} k^{\lambda}$ versus $k^{\infty}$

\section{Numerics}

In this section, we present some numerical results which illustrate the asymptotic relations between the intermediate case (PTPM) and the limiting cases (VTPM and HTPM). All numerical computations were done in COMSOL multiphysics ('creeping flow' module) which is a software based on the finite element method. The geometries $\mathbf{Q}^{f}$ and $Q^{f}$ (see Fig. 3) are divided into triangular mesh elements of variable size. The mesh was refined successively until we obtain the required convergence. In the computations presented below, we used 12,570 elements for the $3 D$ cell problems and 762 elements for the $2 D$ problems.

In particular, it is shown that $K^{\lambda} \sim K^{0}$ for small values of $\lambda$ and that $K^{\lambda} \sim \lambda^{-2} K^{\infty}$ for large values of $\lambda$. Recall that cell problems to compute permeability depend only on the dimensionless parameter $\lambda$ and the radius $R$ of the cylinder inclusions. Thus we can regard $K^{0}$ and $K^{\infty}$ as functions of $R$ only. For this particular geometry, the permeability tensors are described by single scalars $k^{\lambda}, k^{0}$ and $k^{\infty}$ (see (16), (28) and (38)) in all three cases, respectively.

Solving the PTPM cell problem (12) in the domain $\boldsymbol{Q}^{f}$ for different radii $R=$ $0.1,0,2,0.3,0.4$ and different $\lambda \in\left[2^{-8}, 2^{4}\right]$ allows us to compute permeability as a function of $R$ and $\lambda$. In order to compare $k^{\lambda}$ with the limit cases $\left(k^{0}\right.$ and $\left.k^{\infty}\right)$, we also solve the problems (43) and (26).

In view of (37) $k^{\lambda} \sim k^{\infty} / \lambda^{2}$, for the HTPM-case. Figure 4 shows $\lambda^{2} k^{\lambda}$ as a function of $\lambda$ for various fixed $R$, where the dotted lines correspond to $k^{\infty}$ which is a function of $R$ alone. This suggests that $k^{\infty}$ may be used as a good approximation for $\lambda^{2} k^{\lambda}$ for large values of $\lambda$ as shown in the above analysis. The relative error in this HTPM-approximation is displayed in Table 4. It can be observed that the convergence seems faster for larger values of $R$. 
Table $4 k^{\infty}$ versus $\lambda^{2} k^{\lambda}$

\begin{tabular}{llccc}
\hline$R$ & 0.1 & 0.2 & 0.3 & 0.4 \\
\hline 2 & $\left(k^{\infty}-\lambda^{2} k^{\lambda}\right) / k^{\infty}($ relative error, \%) & \\
\hline 4 & 30.9 & 22.3 & 15.4 & 8.2 \\
6 & 15.6 & 11.2 & 7.7 & 4.4 \\
8 & 10.4 & 7.6 & 5.1 & 2.7 \\
10 & 7.8 & 5.6 & 3.8 & 2.2 \\
12 & 6.3 & 4.5 & 3.1 & 1.6 \\
14 & 5.3 & 3.8 & 2.6 & 1.6 \\
16 & 4.5 & 3.3 & 2.3 & 1.6 \\
\hline
\end{tabular}

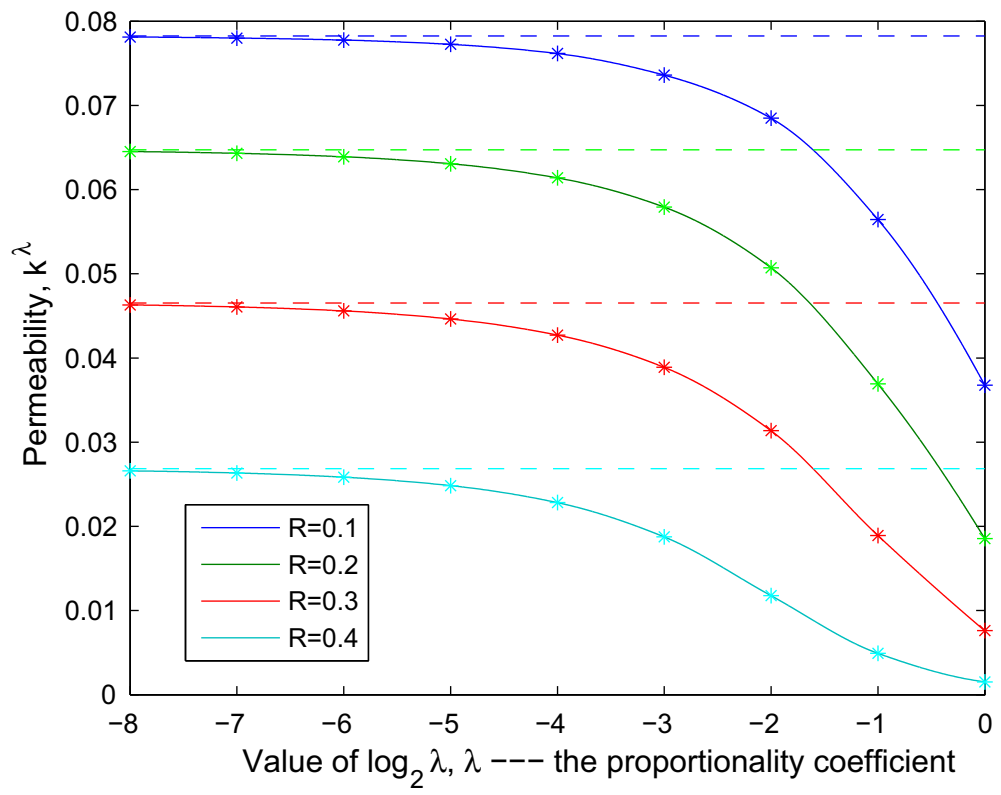

Fig. $5 k^{\lambda}$ versus $k^{0}$

In view of (27) $k^{\lambda} \sim k^{0}$, for the VTPM-case. Figure 5 shows $k^{\lambda}$ as a function of $\lambda$ for various fixed $R$, where the dotted lines correspond to $k^{0}$ which is a function of $R$ alone. Here a logarithmical scale is used for the $\lambda$-axis. This suggests that $k^{0}$ may be used as a good approximation for $k^{\lambda}$ for small values of $\lambda$ as shown in the above analysis. The relative error in this VTPM-approximation is displayed in Table 5. Here it can be observed that the convergence seems faster for smaller values of $R$, as opposed to the HTPM-case.

In the limit cases, $k^{\infty}$ and $k^{0}$ are functions that only depend on $R$ (the micro geometry). These dependencies are shown in Figs. 6 and 7, respectively. 
Table $5 k^{0}$ versus $k^{\lambda}$

\begin{tabular}{lcccc}
\hline$R$ & 0.1 & 0.2 & 0.3 & 0.4 \\
$\lambda$ & $\left(k^{0}-k^{\lambda}\right) / k^{0}($ relative error, \%) & & 56.1 \\
\hline $2^{-2}$ & 12.5 & 21.6 & 32.6 & 30.1 \\
$2^{-3}$ & 6.0 & 10.5 & 16.4 & 15.0 \\
$2^{-4}$ & 2.7 & 5.1 & 8.2 & 7.5 \\
$2^{-5}$ & 1.3 & 2.5 & 4.1 & 3.7 \\
$2^{-6}$ & 0.6 & 1.3 & 2.0 & 1.9 \\
$2^{-7}$ & 0.3 & 0.6 & 1.0 & 0.9 \\
$2^{-8}$ & 0.2 & 0.3 & 0.5 & \\
\hline
\end{tabular}

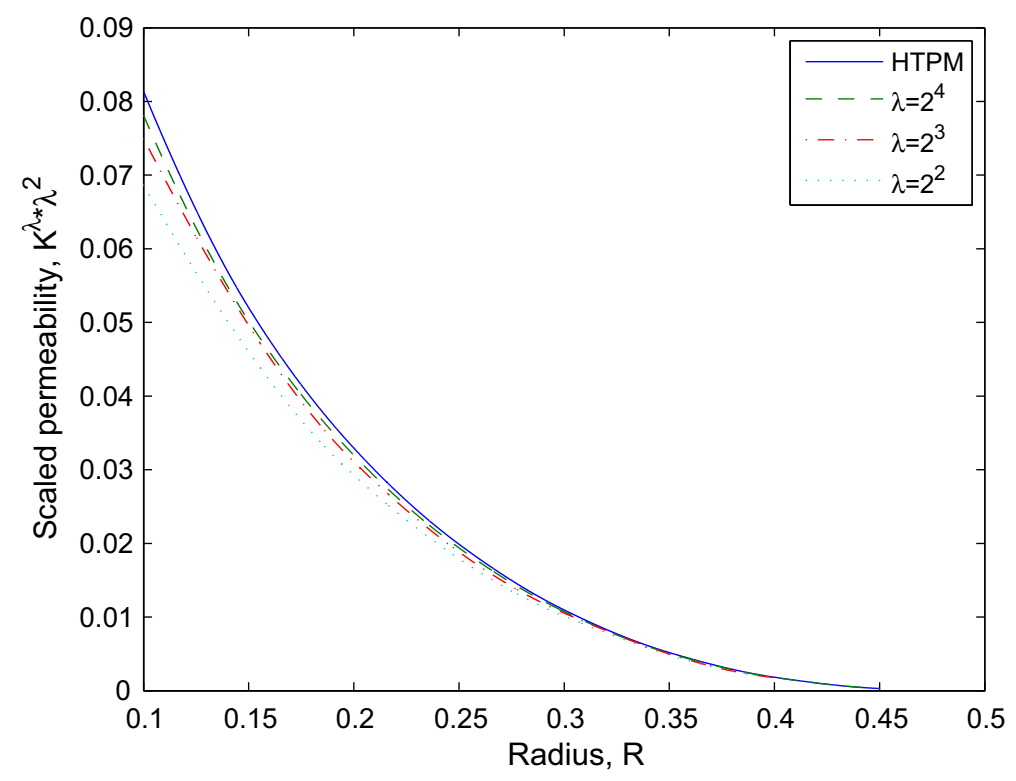

Fig. $6 \lambda^{2} k^{\lambda}(R)$ versus $k^{\infty}(R)$

\section{Conclusions}

Summing up, we have considered flow in a thin porous medium with two small parameters $\varepsilon$ and $\delta$, related to the microstructure and the thickness of the domain. Letting $\varepsilon$ and $\delta$ tend to zero at different rates, asymptotic analysis leads to the following results.

For PTPM, VTPM and HTPM, the flow is governed by equations (13), (41) and (44) correspondingly. These equations are two-dimensional versions of Darcy's law (third components in all equations vanish). We therefore regard $K^{\lambda}, K^{0}$ and $K^{\infty}$ as $2 D$ tensors throughout this section. The asymptotic behaviour of the flow can be described by the diagrams shown in Figs. 8, 9, 10. In Fig. 9, $p^{\lambda}, 0 \leq \lambda \leq \infty$, is the solution of the well-known $2 D$ Darcy equation 


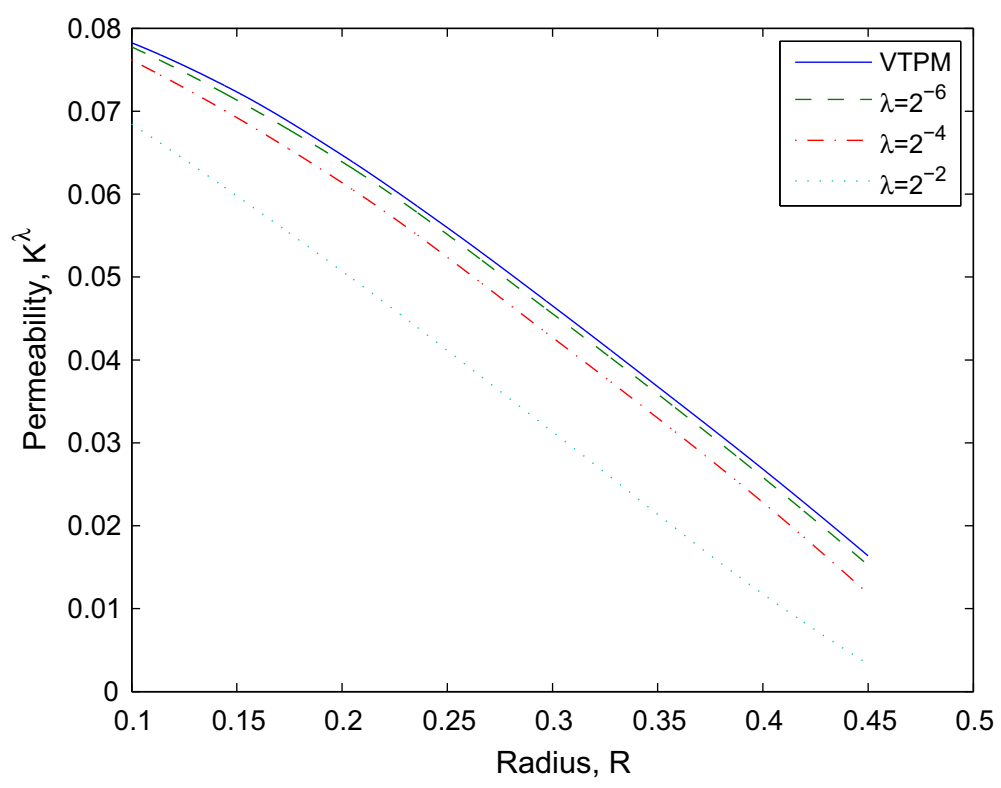

Fig. $7 k^{0}(R)$ versus $k^{\lambda}(R)$

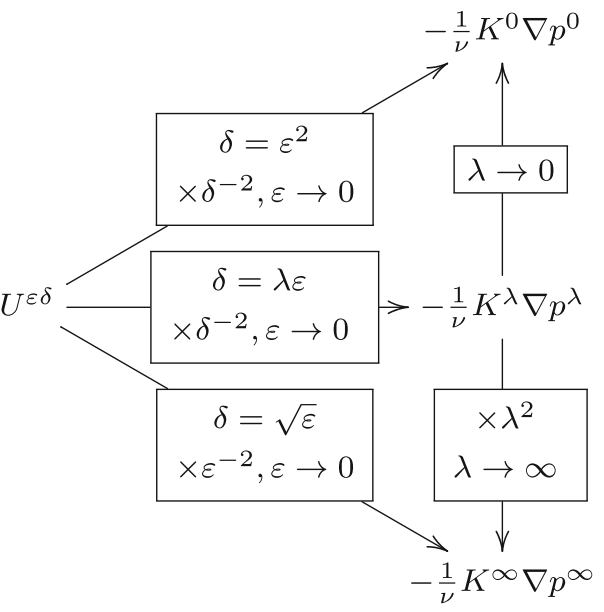

Fig. 8 Asymptotic behaviour of $U^{\varepsilon \delta}$

$$
\begin{cases}\nabla \cdot\left(K^{\lambda} \nabla p^{\lambda}\right)=0 & \text { in } \Omega \\ p^{\lambda}=p^{b} & \text { on } \partial \Omega\end{cases}
$$

Observe that the Dirichlet boundary condition on $\partial \Omega$ in (45) corresponds to the Neumann boundary condition on $\Gamma^{\varepsilon \delta}$ in (2). The reverse also holds, i.e. a Dirichlet boundary condition on $\Gamma^{\varepsilon \delta}$ in the original problem would imply a Neumann boundary condition in the Darcy law. Although (45) holds in all three cases, the permeability is fundamentally different in the limiting cases $\lambda=0$ and $\lambda=\infty$ compared to the intermediate case $0<\lambda<\infty$. More precisely, 


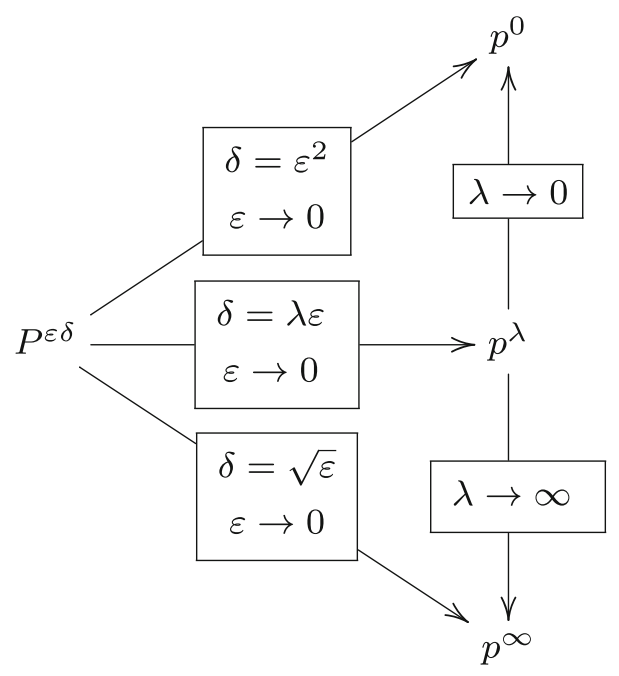

Fig. 9 Asymptotic behaviour of $P^{\varepsilon \delta}$

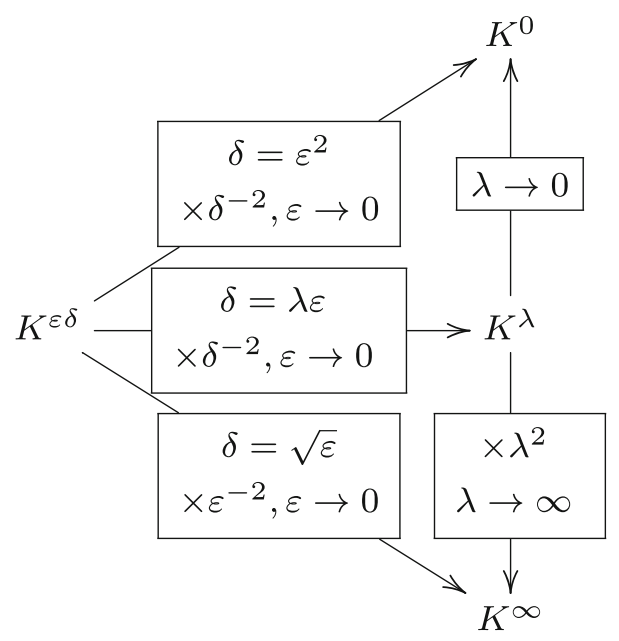

Fig. 10 Asymptotic behaviour of $K^{\varepsilon \delta}$

- Darcy's law for VTPM can be obtained as an asymptotic limit in two different ways (see flow diagram). This is because the local problems (26) and (40) to compute $K^{0}$ are identical. Here, the permeability is given by

$$
K^{0}=-\frac{1}{12|Q|} \int_{Q^{f}}\left(\begin{array}{cc}
\frac{\partial q^{1}}{\partial y_{1}}+1 & \frac{\partial q^{2}}{\partial y_{1}} \\
\frac{\partial q^{1}}{\partial y_{2}} & \frac{\partial q^{2}}{\partial y_{2}}+1
\end{array}\right) \mathrm{d} y,
$$

where $q^{i}$ are solutions of the Hele-Shaw type $2 D$ cell problems

$$
\left\{\begin{array}{ll}
\Delta q^{i}=0 & \text { in } Q^{f} \\
\left(\nabla q^{i}+e^{i}\right) \cdot \boldsymbol{n}=0 & \text { on } S \\
q^{i} & Q \text {-periodic, }
\end{array} \quad(i=1,2)\right.
$$


where $e^{1}=(1,0)$ and $e^{2}=(0,1)$.

- Darcy's law for PTPM is obtained by assuming $\delta=\lambda \varepsilon$ (see flow diagram). Here, the permeability is given by

$$
K^{\lambda}=\frac{1}{|\boldsymbol{Q}|} \int_{\boldsymbol{Q}^{f}}\left(\begin{array}{ll}
W_{1}^{1} & W_{1}^{2} \\
W_{2}^{1} & W_{2}^{2}
\end{array}\right) \mathrm{d} y \mathrm{~d} z,
$$

where $\left(W^{i}, q^{i}\right)$ are solutions of the $3 D$ Stokes cell problems

$$
\left\{\begin{array}{ll}
-\frac{1}{\lambda} \nabla_{\lambda} q^{i}+\Delta_{\lambda} W^{i}-\frac{1}{\lambda^{2}} e^{i}=0 & \text { in } \boldsymbol{Q}^{f} \\
\nabla_{\lambda} \cdot W^{i}=0 & \text { in } \boldsymbol{Q}^{f} \\
W^{i}=0 & \text { on } \boldsymbol{S} \\
W^{i}, q^{i} & \text { Q-periodic, }
\end{array} \quad(i=1,2)\right.
$$

where $e^{1}=(1,0,0)$ and $e^{2}=(0,1,0)$.

- Darcy's law for HTPM can also be obtained as an asymptotic limit in two different ways, as the local problems (36) and (43) to compute $K^{\infty}$ are identical. Here, the permeability is given by

$$
K^{\infty}=\frac{1}{|Q|} \int_{Q^{f}}\left(\begin{array}{ll}
W_{1}^{1} & W_{1}^{2} \\
W_{2}^{1} & W_{2}^{2}
\end{array}\right) \mathrm{d} y,
$$

where $\left(W^{i}, q^{i}\right)$ are solutions of the $2 D$ Stokes cell problems

$$
\left\{\begin{array}{ll}
-\nabla q^{i}+\Delta W^{i}-e^{i}=0 & \text { in } Q^{f} \\
\nabla \cdot W^{i}=0 & \text { in } Q^{f} \\
W^{i}=0 & \text { on } S \\
W^{i}, q^{i} & Q \text {-periodic, }
\end{array} \quad(i=1,2)\right.
$$

where $e^{1}=(1,0)$ and $e^{2}=(0,1)$.

From an engineering point of view, the present analysis shows that the two-dimensional approaches to computing the permeability of thin porous media must be used carefully. As given in Tables 4 and 5, the error can be substantial if one uses the $2 D$ cell problems (47) or (51) instead of (49). Hence it is important to distinguish between three kinds of porous media, namely VTPM, PTPM and HTPM.

Open Access This article is distributed under the terms of the Creative Commons Attribution 4.0 International License (http://creativecommons.org/licenses/by/4.0/), which permits unrestricted use, distribution, and reproduction in any medium, provided you give appropriate credit to the original author(s) and the source, provide a link to the Creative Commons license, and indicate if changes were made.

\section{References}

Allaire, G.: Homogenization of the Stokes flow in a connected porous medium. Asymptot. Anal. 2, 203-222 (1989)

Batchelor, G.K.: An Introduction to Fluid Dynamics. Cambridge University Press, Cambridge (1967)

Chen, X., Papathanasiou, D.: The transverse permeability of disordered fiber arrays: a statistical correlation in terms of the mean nearest interfiber spacing. Transp. Porous Med. 71, 233-251 (2008)

Frishfelds, V., Lundström, T.S., Jakovics, A.: Lattice gas analysis of liquid front in non-crimp fabrics. Transp. Porous Med. 84, 75-93 (2011)

Gebart, B.R.: Permeability of unidirectional reinforcements for RTM. J. Compos. Mater. 26, 1100-1133 (1992)

Hellström, J.G.I., Frishfelds, V., Lundström, T.S.: Mechanisms of flow induced deformation of porous media. J. Fluid Mech. 664, 220-237 (2010) 
Hellström, J.G.I., Jonsson, P.J.P., Lundström, T.S.: Laminar and turbulent flow through an array of cylinders. J. Porous Media 13, 1073-1085 (2010)

Hornung, U.: Homogenization and Porous Media. Springer, New York (1997)

Hwang, W.R., Advani, S.G.: Numerical simulations of Stokes-Brinkman equations for permeability prediction of dual-scale fibrous porous media. Phys. Fluids 22, 113101 (2010)

Jeon, W., Shin, C.B.: Design and simulation of passive mixing in microfluidic systems with geometric variations. Chem. Eng. J. 152, 575-582 (2009)

Jourak, A., Frishfelds, V., Lundström, T.S., Herrmann, I., Hedström, A.: The calculations of dispersion coefficients inside two-dimensional randomly packed beds of circular particles. AIChE J. 59, 1002-1011 (2013)

Koch, D.L., Ladd, A.J.C.: Moderate Reynolds number flows through periodic and random arrays of aligned cylinders. J. Fluid Mech. 349, 31-66 (1997)

Lions, J.-L.: Some Methods in the Mathematical Analysis of Systems and Their Control. Science Press and Gordon and Breach, Beijing (1981)

Lundström, T.S., Gebart, B.R.: Effect of perturbation of fibre architecture on permeability inside fibre tows. J. Compos. Mater. 29, 424-443 (1995)

Lundström, T.S., Toll, S., Håkanson, J.M.: Measurements of the permeability tensor of compressed fibre beds. Transp. Porous Med. 47, 363-380 (2002)

Lee, J.S., Fung, Y.C.: Stokes flow around a circular cylindrical post confined between two parallel plates. J. Fluid Mech. 37, 657-670 (1969)

Nordlund, M., Lundström, T.S.: Effect of multi-scale porosity in local permeability modelling of non-crimp fabrics. Transp. Porous Med. 73, 109-124 (2008)

Saffman, P.G., Taylor, G.: The penetration of a fluid into a porous medium or Hele-Shaw cell containing a more viscous liquid. Proc. R. Soc. Ser. A 245, 312-329 (1958)

Sanchez-Palencia, E.: Non-homogeneous Media and Vibration Theory, Lecture Notes in Physics, vol. 129. Springer, Berlin (1980)

Sangani, A.S., Yao, C.: Transport processes in random arrays of cylinders. II. Viscous flow. Phys. Fluids 31, 2435-2444 (1988)

Sherman, F.S.: Viscous flow. McGraw-Hill International Editions, Singapore (1990)

Singh, F., Stoeber, B., Green, S.I.: Micro-PIV measurement of flow upstream of papermaking forming fabrics. Transp. Porous Med. 107, 435-448 (2015)

Tan, H., Pillai, K.M.: Multiscale modeling of unsaturated flow in dual-scale fiber preforms of liquid composite molding I: Isothermal flows. Compos. Part A Appl. Sci. Manuf. 43, 1-13 (2012)

Tartar, L.: Incompressible Fluid Flow in a Porous Medium-Convergence of the Homogenization Process, Lecture Notes in Physics, vol. 129, pp. 368-377. Springer, Berlin (1980)

Taylor, G.: Film notes for low-Reynolds-number flows, No. 21617, National Committee for Fluid Mechanics Films, Encyclopedia Britannica Educational Corporation, Chicago (1967)

Tsay, R.-Y., Weinbaum, S.: Viscous flow in a channel with periodic cross-bridging fibres: exact solutions and Brinkman approximation. J. Fluid Mech. 226, 125-148 (1991)

Van Dyke, M.: An Album of Fluid Motion. The Parabolic Press, Stanford (1982)

Whitaker, S.: Flow in porous media I: a theoretical derivation of Darcy's law. Transp. Porous Media 1, 3-25 (1986) 